La Revue

des Droits

de l'Homme

\section{La Revue des droits de l'homme}

Revue du Centre de recherches et d'études sur les droits fondamentaux

Actualités Droits-Libertés | 2017

\title{
Les lieux privatifs de liberté en Italie : de la Cour constitutionnelle au Comité européen pour la prévention de la torture
}

Droit pénitentiaire (Italie)

Yéris Nicolas et Margaux Romano

\section{(2) OpenEdition}

Journals

Édition électronique

URL : http://journals.openedition.org/revdh/3327

DOI : $10.4000 /$ revdh.3327

ISSN : 2264-119X

Éditeur

Centre de recherches et d'études sur les droits fondamentaux

Référence électronique

Yéris Nicolas et Margaux Romano, « Les lieux privatifs de liberté en Italie : de la Cour constitutionnelle au Comité européen pour la prévention de la torture », La Revue des droits de l'homme [En ligne], Actualités Droits-Libertés, mis en ligne le 30 octobre 2017, consulté le 01 mai 2019. URL : http:// journals.openedition.org/revdh/3327 ; DOI : 10.4000/revdh.3327

Ce document a été généré automatiquement le 1 mai 2019.

Tous droits réservés 


\title{
Les lieux privatifs de liberté en Italie : de la Cour constitutionnelle au Comité européen pour la prévention de la torture
}

\author{
Droit pénitentiaire (Italie)
}

Yéris Nicolas et Margaux Romano

1 Peu nombreux sont les États où les prisons sont irréprochables, et l'Italie n'en fait vraisemblablement pas partie. Malgré plusieurs décisions rendues au premier semestre 2017 par la Cour constitutionnelle italienne au sujet des conditions de détention dans ses prisons $^{1}$, l'Italie est régulièrement pointée du doigt, et parfois condamnée, par les instances européennes. Dans un pays où la surpopulation carcérale constitue un enjeu essentiel, les moyens pour y faire face ne sont malheureusement pas toujours à la hauteur des attentes nationales et internationales.

2 Les conditions de détention au sein des prisons italiennes ont fait l'objet de plusieurs décisions de la Cour constitutionnelle en 2017. Celles-ci s'ajoutent aux multiples décisions de la Cour européenne des droits de l'homme (Cour EDH) ${ }^{2}$, et au rapport du Comité européen pour la prévention de la torture (CPT) rendu public le 8 septembre 2017², lesquels se révèlent assez virulents à l'égard de la législation nationale. De la lecture de ces documents, il ressort avant tout que la surpopulation carcérale entraine des conditions de détention s'apparentant à des traitements inhumains et dégradants (I). Ensuite, il est relevé que les relations avec les proches sont souvent difficiles, si ce n'est impossibles (II) et que la prise en charge des détenus à la santé fragile est encore trop faible (III). Enfin, les droits procéduraux ne sont pas suffisamment garantis (IV). Les efforts entrepris par l'Italie pour surmonter cette crise semblent, de manière générale, privés d'efficacité réelle. 


\section{1 - Traitements inhumains et dégradants}

\section{A - La surpopulation carcérale}

On compte en Italie 194 prisons, dont 131 sont en situation de surpopulation carcérale, à savoir près de $68 \%$. Cela correspond à 10000 personnes incarcérées au-delà du nombre de places prévues dans les prisons italiennes. Ce chiffre est alarmant. La sonnette d'alarme avait été tirée par la Cour européenne des droits de l'homme au moins à deux reprises. L'Italie avait notamment été condamnée par la Cour dans l'arrêt Sulejmanovic c. Italie du 16 juillet2009. Le gouvernement italien réagit en déclarant un stato di emergenza par décret du 13 janvier 2010 pour faire face à la surpopulation carcérale. Cette mesure exceptionnelle, prise en vertu de la loi no 225 de 1992 sur la protection civile, avait permis au gouvernement de prendre des mesures d'urgence en accordant des pouvoirs exceptionnels au chef du département de l'administration pénitentiaire. La construction de 18 prisons sur le territoire italien avait également été entreprise. Ce décret avait aussi permis aux personnes condamnées à des peines de prison inférieures à 12 ans d'effectuer leur condamnation à domicile. Enfin, avait été prévue la création de 2000 postes d'agents supplémentaires au sein des prisons.

Pourtant, ces mesures ont été jugées insuffisantes par le juge européen dans l'arrêt Torreggiani et autres $c$. Italie. En l'espèce, les requérants affirmaient avoir occupé des cellules de $9 \mathrm{~m}^{2}$ qu'ils partageaient avec deux autres détenus et se plaignaient de manquer d'eau chaude ainsi que, pour certains, d'éclairage suffisant dans leurs cellules. La Cour, après avoir constaté le surpeuplement carcéral au sein des prisons italiennes et les diverses carences matérielles qui en découlent, relève en outre le manque de recours effectif en droit interne pour faire face à ces situations. La Cour considère que cette situation conduit inévitablement à une violation de l'art. 3 de la CEDH qui interdit la torture et les traitements inhumains ou dégradants. En conséquence, elle enjoint le gouvernement italien à « mettre en place un recours ou une combinaison de recours ayant des effets préventifs et compensatoires et garantissant réellement une réparation effective des violations de la Convention résultant du surpeuplement carcéral en Italie » dans un délai d'un an à compter de la date où l'arrêt sera devenu définitif. La Cour a en l'occurrence décidé de répondre par un arrêt pilote, ce qui lui permet de geler les demandes similaires le temps que le gouvernement italien prenne les mesures adéquates.

5 Cependant, la situation reste aujourd'hui encore critique. Début septembre, des détenus de la prison de San Vittore à Milan ont décidé de protester contre cette surpopulation carcérale en refusant de participer aux activités proposées dans le cadre de leur traitement (théâtre, cours d'histoire de l'art, etc.), la plupart faisant face à des problèmes de drogue et d'alcool. Ce manque de places au sein des prisons italiennes est un problème majeur qui érige l'Italie parmi les pays européens disposant d'un taux de surpopulation carcérale extrêmement élevé. Telle est la raison pour laquelle, le Comité européen pour la prévention de la torture (CPT) a interpellé l'Italie dans un rapport publié le 8 septembre 2017, lui rappelant qu'elle se devait de respecter les standards qu'il a fixé en matière d'espace de vie dont doit disposer chaque détenu - soit $6 \mathrm{~m}^{2}$ minimum pour les cellules individuelles et $4 \mathrm{~m}^{2}$ pour les cellules collectives, sans compter les espaces sanitaires. En réponse à ce rapport, le gouvernement italien a affirmé étudier plusieurs possibilités afin de diminuer le nombre de prisonniers, notamment en permettant aux détenus de 
nationalité étrangère de purger leur peine dans leur pays ou en ayant recours plus fréquemment à des mesures alternatives à la détention.

\section{B - Surpopulation et toxicomanie}

6 Cette surpopulation pose des problèmes exacerbés en matière de toxicomanie. La Cour constitutionnelle s'est prononcée à ce sujet dans une décision du 7 juin 2017 portant sur le décret $n^{\circ} 309$ du 9 octobre 1990 relatif aux stupéfiants et substances psychotiques, prévention, soin et réhabilitation pour les individus en état de toxicodépendance. En l'espèce, était contesté le peu de marge de manœuvre laissé au pouvoir souverain du juge et le fait que la peine imposée aux toxicomanes serait contraire à l'obligation de traitements adéquats et à la fonction rééducative d'une détention. Surtout, il était rappelé que le surpeuplement dans les milieux carcéraux, abondamment critiqué, aggrave les risques et les cas de toxicomanie.

7 Pour autant, la Cour constitutionnelle retient qu'un décret du 20 mars 2014, a mis en œuvre des dispositions urgentes pour lutter contre la surpopulation. À cet effet, le plafond des peines carcérales varie du simple au double selon qu'est concernée une addiction aux drogues «dures » ou aux drogues «douces ». Le plafond est de huit ans dans le premier cas et de quatre dans le second. L'interdiction de traitements inhumains consacrée par la Chartre des droits fondamentaux de l'Union européenne ainsi que la Convention EDH est alors respectée selon la Cour.

\section{C - Les fréquentes accusations de mauvais traitements physiques}

Outre la surpopulation carcérale, l'Italie doit faire face à des problèmes de violences au sein de ses prisons. Le CPT a signalé des cas de mauvais traitements physiques infligés non seulement aux personnes privées de liberté par les forces de l'ordre mais aussi envers des personnes incarcérées. Le rapport souligne notamment des violences telles que « des coups de poing, des claques, des coups de pied et des coups de matraque ». Il a été constaté que ces violences suivaient généralement des épisodes impliquant des agressions, tentatives de suicide ou suicides de détenus. Pour cette raison, le CPT recommande aux autorités italiennes de dispenser une formation au personnel pénitentiaire afin de lui permettre de faire face à ces situations sans violence. Il souligne également qu'il est impératif de rappeler aux forces de l'ordre que les violences physiques sont inacceptables ainsi que les sanctions encourues en cas de mauvais traitement physique avéré.

Mention spéciale doit également être faite du régime spécifique prévu à l'article 41 bis de la loi du 26 juillet $1975 n^{\circ} 354$ sur le système pénitencier. Celui-ci est un des plus controversés de la législation pénitentiaire italienne mais aussi un des plus importants. Cette disposition prévoit un régime carcéral spécial pour les détenus ayant été condamnés pour des crimes en lien avec la criminalité organisée ou le terrorisme. Le régime consiste notamment à isoler le détenu des autres personnes incarcérées, à limiter ses heures de promenade, à limiter les rencontres avec les proches (une par mois) ainsi qu'avec les avocats, à contrôler strictement la correspondance ou encore à interdire de disposer de plus d'un certain nombre d'objets dans la cellule. On peut se demander dans quelle mesure ce régime est compatible tant avec les exigences des droits et libertés de la Constitution italienne qu'avec celles de la Convention EDH. 
10 La Cour constitutionnelle italienne a eu à se prononcer sur la constitutionnalité de cet article dans une décision $n^{\circ} 122$ du 8 février 2017. En l'espèce, une circulaire du 16 novembre $2011 \mathrm{n}^{\circ} 8845$, se fondant sur l'art. 41 bis, prévoyait un contrôle strict des envois et des réceptions de livres, revues et quotidiens aux détenus soumis au régime spécial. En particulier, les détenus ne pouvaient se procurer ces biens qu'auprès de l'institut pénitencier, impliquant de fait l'interdiction de se les procurer par colis. Cette disposition visait notamment à éviter que les livres, revues et quotidiens deviennent des supports de communication avec les personnes extérieures, alors même que cette communication est limitée dans le cadre du régime carcéral spécial. Il s'agissait donc de savoir si cette restriction n'était pas contraire à la liberté de correspondance (art. 15 de la constitution), à la liberté de pensée entendue comme droit à l'information et droit à l'enseignement (art. 21, 33 et 34 de la Constitution) ainsi qu'aux engagements internationaux pris par l'Italie (art. 117 de la Constitution), notamment les articles 3 et 8 de la Convention EDH.

11 S'agissant de la présumée violation de la liberté de correspondance, les juges suprêmes retiennent que la question n'est pas fondée étant donné que la restriction ne concerne pas spécifiquement les publications, mais plus généralement tout échange d'objet qui pourrait constituer un moyen de communication avec l'extérieur. De même, la question relative à la liberté d'information et au droit à l'enseignement est jugée infondée. La Cour retient que la faculté des détenus de choisir les textes par lesquels s'informer et s'instruire n'est en rien limitée, seules les modalités par lesquelles ils peuvent se procurer ces documents le sont. Enfin, la Cour constitutionnelle considère que cette disposition est pleinement conforme à la Convention EDH puisque la limitation opérée de l'échange des biens avec l'extérieur est proportionnée et n'entache pas le droit à la vie privée et familiale. Si la Cour ne semble rien avoir à reprocher à la législation relative aux conditions de détention des prisonniers soumis au régime de l'art. 41 bis, le CPT, dans le rapport précité de septembre 2017, semble pour sa part beaucoup plus sceptique. Il relève notamment que la surveillance vidéo est permanente et que, excepté l'enseignement à distance, aucune autre activité n'est proposée à ces détenus. Le CPT s'interroge également sur la mesure visant à limiter le nombre d'objets dans les cellules, celle-ci pourrait conduire à des cas de mauvais traitement. Le CPT prend pour exemple le cas d'un détenu soumis à l'art. 41 bis qui souffrait d'une allergie et qui s'était vu prescrire une couverture en coton en 2014. La direction de la prison a toujours refusé de lui faire parvenir cette couverture, se prévalant de de sa faculté légale de limiter les objets dans les cellules. Au moment du passage du CPT en avril 2016, le détenu en question n'avait toujours pas reçu ladite couverture lui permettant d'atténuer son allergie. Lors de ses précédents rapports, le CPT avait déjà signalé que les mesures prises dans le cadre de l'art. 41 bis et appliquées sur une durée prolongée pouvaient constituer un traitement inhumain et dégradant.

\section{2 - L'autorité parentale en milieu carcéral}

12 Alors que le système judiciaire est en principe fondé sur l'individualité des peines, lorsqu'une mère ou un père va en prison, leurs enfants sont aussi affectés. Ne pas considérer la situation des enfants de parents emprisonnés au long de toutes les étapes de la procédure criminelle - de l'arrestation, en passant par le procès, l'emprisonnement, la libération, à la réintégration au sein de la communauté - risque de porter atteinte à leurs droits, leurs besoins et leurs intérêts supérieurs. Or, il n'existe en Italie qu'un nombre limité de facteurs susceptibles d'infléchir la peine prononcée : les besoins ou les intérêts 
des enfants affectés sont souvent peu analysés. Un progrès est néanmoins perceptible pour les femmes enceintes ou pour les mères de nouveau-nés. La loi du 21 avril 2011 interdit ainsi d'emprisonner une femme enceinte en Italie. Dans les autres cas, le système pénitencier italien rencontre de nombreux problèmes au sujet des mères en milieu carcéral. La Cour constitutionnelle s'est exprimée à ce sujet dans deux récentes décisions.

13 La première décision, en date du 7 décembre 2016, est relative à l'article 275 du Code de procédure pénale. Celui-ci impose la détention à domicile pour tout détenu condamné pour participation à une organisation criminelle, dès lors que leur enfant est âgé de moins de 6 ans. En l'espèce, une mère et un père d'un enfant mineur avaient été condamnés pour association à une organisation criminelle. La mère était détenue à domicile en raison de l'âge de leur enfant. En novembre 2015, l'enfant atteignit l'âge de six ans. En application de l'article 275 du Code de procédure pénale, la mère était alors contrainte de finir de purger sa peine dans un établissement pénitencier. Il était soutenu devant la Cour que, privée de toute appréciation concrète de la situation particulière de l'enfant, l'application automatique de cette norme était contraire aux principes constitutionnels italiens. L'inconstitutionnalité de l'article 275 du Code de procédure pénale fut ainsi soulevée sur le fondement de deux principes constitutionnels : le principe de proportionnalité et de non automaticité des peines (art. 3 de la Constitution) ainsi que le droit à une vie de famille normale doublée de la considération de l'intérêt supérieur de l'enfant (art. 31 de la Constitution et Convention de New York de 1989 relative aux droits de l'enfant).

14 La Cour considère que la détention à domicile subordonnée à l'âge de l'enfant doit répondre à un équilibre entre l'exigence, d'une part, de protection de l'enfant mineur et, d'autre part de protection de l'intérêt général. Selon la Cour constitutionnelle, toute peine prononcée est prise en considération des circonstances atténuantes ou aggravantes spécifiques au délit. Or, l'enfant est tiers au procès et extérieur à l'acte frauduleux. L'âge de l'enfant est alors un critère objectif qui n'est pas susceptible d'influencer l'application de la peine prononcée. Le recours fut en conséquence rejeté.

La seconde décision, du 8 mars 2017, porte sur l'article 47 de la loi n³54 du 26 août 1975 . Celui-ci prévoit qu'une condamnation à trois ans d'emprisonnement ferme peut être commuée en peine de substitution. La mère exécutera alors sa peine à domicile suivie par les services sociaux. Néanmoins, cet article s'applique en cas de peines spécifiques subordonnées à l'âge de l'enfant. En l'espèce, la mère avait été condamnée à sept ans de prison sur le fondement de la loi relative à l'usage de stupéfiants et substances psychotiques, préventions, soins et réhabilitations. Cette peine avait été convertie en détention à domicile jusqu'au troisième anniversaire de l'enfant. Elle demandait la prorogation de la détention à domicile sur le fondement du principe de l'intérêt supérieur de l'enfant et du droit à une vie de famille normale. Le tribunal de Bari a alors soulevé la question de constitutionnalité de l'article 47 de la loi du 26 août 1975 au regard des articles 3, 29, 30 et 31 de la constitution.

La Cour rappelle que la loi du 26 août 1975 a pour objectif de faire prévaloir la prévention et la protection de la maternité et de l'enfant mineur, et de sauvegarder le droit à une vie de famille normale. Elle rappelle également, comme dans de nombreuses autres décisions, la prééminence de l'intérêt supérieur de l'enfant afin qu'il maintienne un lien effectif et continu avec chacun de ses parents, desquels il recevra des soins et une éducation. L'exigence de la protection de la société doit prendre en considération les situations concrètes, l'intérêt supérieur de l'enfant, et non pas appliquer des mécanismes 
automatiques qui empêchent le juge d'apprécier la particularité de chaque situation. Dès lors, la Cour constitutionnelle déclare l'inconstitutionnalité de l'article 47 de la loi s'agissant des termes "sauf à l'égard des mères condamnées pour les délits prévus à l'article 4 bis » de la loi. L'article 4 bis énumère limitativement les délits pour lesquels les mères ne peuvent bénéficier des peines de substitution, en cas de participation à une organisation criminelle, terroriste, ou à un trafic de drogue international.

\section{3 - Les cas particuliers des détenus à la santé fragile}

Le cas des détenus à la santé fragile représente une situation délicate en ce qu'elle repose sur un équilibre précaire entre sécurité et droit à la santé en milieu carcéral.

\section{A - La fermeture des hôpitaux psychiatriques judiciaires et la création des REMS pour maintenir l'accès aux soins}

18 Pendant plusieurs années, les hôpitaux psychiatriques judiciaires italiens ont été au cœur de nombreuses polémiques. L'art. 222 du Code pénal italien prévoit l'hospitalisation en hôpital psychiatrique judiciaire des personnes condamnées pour avoir commis une infraction pénale mais reconnues incapables "d'entendre et de vouloir », selon l'expression italienne consacrée. Dès 2003, dans sa décision $\mathrm{n}^{\circ} 253 \mathrm{du} 18$ juillet, la Cour constitutionnelle déclare inconstitutionnelle une partie de cet article en raison du manque d'alternatives offertes au juge dans de telles situations. En effet, lorsqu'une personne incapable « d'entendre et de vouloir » est condamnée, le juge n'a d'autre choix que de procéder à l'hospitalisation dans ces institutions, et ce même si une mesure moins sévère aurait permis non seulement d'assurer les soins appropriés au patient mais aussi de faire face à sa dangerosité sociale. La Cour considère que l'automatisme de cette mesure est inconstitutionnel puisqu'il conduit à rompre l'équilibre et la proportionnalité que recherche le juge dans l'application des peines.

Même si les professionnels de santé, tout comme les praticiens du droit, avaient connaissance des conditions de vie déplorables qui régnaient dans ces hôpitaux, c'est une commission d'enquête parlementaire qui, en 2011, dévoile au grand jour cette triste réalité. Le rapport évoque notamment des carences matérielles graves et inacceptables dans tous les hôpitaux psychiatriques judiciaires - lesquels se rapprochent davantage des prisons que des services psychiatriques -, ainsi que l'insuffisance du personnel soignant au sein de ces hôpitaux. Par ailleurs, le recours à la force et la contention physique, s'ils sont certes justifiés dans certains cas selon le CPT, constituent généralement des pratiques inadaptées aux situations et représentent une atteinte à la dignité de la personne.

20 Le décret-loi $n^{\circ} 211$ du 22 décembre 2011, modifié et converti par la loi nº 9 du 17 février 2012, prévoit à l'art. 3 ter, la fermeture définitive des hôpitaux psychiatriques judiciaires avant le 31 mars 2013. Ce terme a été repoussé, d'abord au 31 mars 2014, puis finalement au 31 mars 2015. Les OPG supprimés ont été remplacés par des Résidences pour l'exécution des mesures de sécurité (REMS), de plus petite envergure. Désormais, les personnes condamnées pour une infraction pénale mais ne pouvant être incarcérées en raison de leur incapacité « d'entendre et de vouloir » seront séparées dans deux structures : les personnes présentant une maladie psychique permanente, socialement dangereuses et 
pour lesquelles il existe un lien direct entre la maladie et l'infraction commise iront dans lesdites REMS; les autres seront prises en charge par les services psychiatriques des hôpitaux. Cette réforme permet ainsi de proportionner la mesure de sécurité à la dangerosité de la personne. Cette initiative a été saluée par la CPT dans son rapport. Toutefois, il met en garde le gouvernement italien concernant la nécessité de ne pas transformer ces REMS en hôpitaux psychiatriques judiciaires au fil du temps.

\section{B - Le manque de places au sein des nouvelles structures gérées par les Régions en raison du manque de moyens financiers alloués}

21 Le nouvel article 3 ter du décret-loi du 22 décembre $2011\left(n^{\circ} 211\right)$ donne du fil à retordre à la Cour constitutionnelle italienne. Dans sa décision n ${ }^{\circ} 22$ du 26 janvier 2017, celle-ci s'est interrogée sur sa constitutionnalité, notamment au regard de l'art. 3 de la Constitution italienne qui pose le principe de l'égalité de tous les citoyens face à la loi. En l'espèce, le procès d'une personne avait été suspendu en raison de son incapacité «d'entendre et de vouloir ", ce qui avait conduit à son placement au sein d'une résidence pour l'exécution des mesures dites de "sécurité ». L'art. 3 ter dispose que cette mesure, qu'elle soit définitive ou provisoire, ne doit pas s'étendre au-delà de la durée de la peine maximale de réclusion prévue pour l'infraction commise. Le juge du fond avait considéré que l'art. 3 ter était contraire au principe d'égalité consacré par la Constitution italienne en ce qu'il faisait coïncider la fin de la mesure de sécurité avec la peine maximale prévue pour l'infraction plutôt qu'avec la cessation de la dangerosité de l'individu. Par ailleurs, le juge rappelait que la détention provisoire et la mesure de sécurité n'ont pas la même fonction : la première vise à éviter la dégradation des éléments de preuve, prévenir la fuite ou la récidive des infractions, là où la seconde vise à soigner la personne d'une maladie qui la rendrait socialement dangereuse.

La Cour constitutionnelle rejette néanmoins la demande. Elle rappelle que l'art. 3 ter tend à éviter des situations dites de "perpétuité blanche ", qui conduisent à placer des individus au sein d'établissements comme les REMS pour une durée indéterminée. Mais elle considère aussi que la situation qui lui est soumise est délicate puisqu'elle conduit à remettre en liberté et sans contrôle, notamment en raison du manque de places au sein des structures spécialisées telles que les REMS, des personnes qui nécessitent des soins et qui sont socialement dangereuses. C'est en effet en partie en raison du manque de places au sein de ces institutions spécialisées que la mesure contenue dans l'art. 3 ter avait été adoptée.

On peut donc encore douter de l'efficacité de cette solution eu égard à l'équilibre tant recherché entre sécurité et droit à la santé en milieu carcéral. Toutefois, il est possible d'espérer une amélioration au cours du temps. Tout d'abord, les REMS ayant été mises en place récemment, un certain recul est nécessaire pour adapter ces institutions aux besoins réels, et plus particulièrement aux besoins des patients. Par ailleurs, les REMS sont désormais gérées par les Régions, ce qui constitue une nouveauté. Il reste que, pour le moment, les moyens financiers nécessaires à leur mise en place n'ont pas forcément suivis cette délégation de compétence. 


\section{4 - Les droits procéduraux}

\section{A - Le secret des correspondances}

24 lequel cède le respect de la vie privée protégée par l'article 8 de la Convention EDH, sous réserve que l'atteinte résultant de la production de la pièce litigieuse soit indispensable à l'exercice de ce droit et proportionnée au but poursuivi. Pour sa part, la Cour constitutionnelle a rappelé dans une décision du 7 décembre 2016 l'encadrement du droit au secret de la correspondance au vu du respect du droit à la preuve. La Cour d'assises d'appel de la Calabre avait en effet soulevé devant la Cour constitutionnelle la violation des articles 3 et 112 de la Constitution par l'article 266 du Code de procédure pénale. Cet article concerne l'admissibilité de la preuve lorsqu'une des parties au procès est un détenu. Il établit une distinction selon que la preuve rapportée est une correspondance épistolaire ou une correspondance par voie de télécommunication. Les correspondances par voie de télécommunication peuvent être interceptées et utilisées lors d'un procès. Quant aux correspondances épistolaires, elles peuvent être seulement mises sous séquestre. Ainsi, le procureur a le pouvoir de priver toute communication épistolaire entre un détenu et des individus extérieurs en imposant la mise sous scellés de toutes lettres provenant d'un condamné.

En l'espèce, il était demandé, sur le fondement du principe d'égalité, que les correspondances épistolaires soient soumises aux mêmes règles que les correspondances par voie de télécommunication. Ceci élargirait le champ des moyens de preuves admissibles afin d'accélérer le processus de recherche de la preuve par l'action publique.

Dans sa décision, la Cour constitutionnelle rappelle que le droit à la liberté, le droit au secret de la correspondance, et par la suite, le principe du contradictoire, peuvent être limités par l'exigence de la protection de la société et par celle de l'efficacité de l'action publique. La restriction de ces droits doit toutefois respecter le principe de proportionnalité. En l'espèce, la Cour estime que la mise sous séquestre des correspondances épistolaires a pour conséquence d'éviter des communications potentiellement dangereuses et qu'elle est justifiée par la nécessité de protéger la société. Aussi, elle rejette la demande et refuse un traitement analogue des deux types de correspondance.

\section{B - Le droit d'accès à un avocat, un droit souvent oublié}

Dans son rapport, le CPT soulève également le problème de l'absence d'information des personnes arrêtées concernant leur droit d'avoir accès à un avocat. Selon l'art. $386 \mathrm{du}$ Code de procédure pénale italien, les officiers et agents de police judiciaire qui ont procédé à une arrestation doivent impérativement informer la personne de ses droits, et notamment de la possibilité de désigner un avocat. Or, souvent, les forces de l'ordre se dispensent de cette obligation. Dans son rapport, le CPT demande aux autorités italiennes que ce droit de la défense soit respecté en toutes circonstances. Il exige qu'une déclaration officielle soit faite aux forces de l'ordre pour le leur rappeler. 


\section{C - La délicate interprétation de la loi quant à l'indemnisation des personnes soumises à des mesures de sûreté privatives de liberté}

L'Italie a toujours été vigilante sur l'obligation de se conformer aux prescriptions de l'Union européenne, notamment lorsqu'elles font suite à des condamnations. Ainsi, après la décision de la Cour européenne des droits de l'homme du 8 janvier 2013, Torreggiani et autres $c$. Italie, elle a décidé de revoir sa législation en matière de recours en cas de violation de la Convention EDH. Il en résulte l'introduction d'un art. 35 ter dans la loi ${ }^{\circ}$ 375 du 26 juillet 1975 par le décret-loi $n^{\circ} 92$ du 26 juin 2016. Celui-ci contient des dispositions urgentes en matière de recours pour les détenus ayant subi un traitement contraire à la Convention EDH. Il prévoit l'introduction d'un recours permettant de demander une réduction de peine et, le cas échéant, une indemnisation financière.

Dans sa décision $n^{\circ} 83$ du 7 mars 2017, la Cour constitutionnelle a été appelée à se demander si ce recours s'appliquait également aux personnes faisant l'objet de mesures de sécurité. En l'espèce, une personne qui avait fait l'objet d'une mesure de sécurité (placement en soin, supra) avait séjourné au moins 132 jours dans une cellule inférieure à $3 \mathrm{~m}^{2}$, contrairement aux standards du CPT, ce qui lui ouvrirait droit soit à une réduction de peine, soit à une indemnité financière. Or, le juge du fond constate que l'art. 35 ter ne fait référence qu'aux détenus et non aux personnes internées. Par ailleurs, comment appliquer une réduction de peine à une mesure de sécurité qui dépend de la dangerosité de l'individu ? En outre, le juge considère que l'indemnité ne peut être accordée que dans les cas où la réduction de peine ne serait pas applicable en raison de la faible durée restant à purger.

La Cour constitutionnelle considère en revanche que l'art. 35 ter s'applique aussi bien aux détenus qu'aux personnes soumises à des mesures de sécurité, comme l'indique le titre de la rubrique qui se réfère aux «individus détenus ou internés ». Selon elle, la réduction de peine peut concerner les mesures de sécurité puisque celles-ci ont une durée maximale, alignée sur la peine maximale encourue pour l'infraction commise. Cependant, elle est consciente que la réduction de peine est difficilement applicable dans ce genre de situation puisque qu'elle dépend uniquement de l'état de santé de la personne qui, de fait, n'est pas établi par avance. L'individu soumis à une mesure de sécurité peut également bénéficier d'une indemnité financière. Sur ce point, la Cour confirme expressément que la réduction de peine et l'indemnité sont exclusives l'une de l'autre. Par conséquent l'individu qui ne peut demander une réduction de peine est habilité à demander la réparation intégrale de son dommage sous forme pécuniaire.

On relèvera que, malgré la volonté de l'Italie de se conformer aux prescriptions de la Cour européenne des droits de l'homme, les mesures mises en place se révèlent non seulement difficiles à interpréter mais aussi difficiles à appliquer. 


\section{Conclusion} l'isolement. Néanmoins, il demande davantage d'encadrement juridique. La même demande est formulée en ce qui concerne le consentement des patients au traitement médical. Dans l'ensemble des REMS visitées, les droits des patients au contact avec le monde extérieur continuent en effet d'être réglementés par les règles du milieu carcéral. Le CPT demande à ce que de nouveaux règlements internes propres à ces établissements soient adoptés. Le CPT relève aussi le cas individuel préoccupant d'un patient de Castiglione delle Stiviere ayant subi de fortes doses de médicaments psychotropes afin d'éviter toute tentative de fuite. Il estime que cette dose pourrait être considérée comme un produit chimique ayant des effets à long terme et appelle l'État italien à proposer des options alternatives plus appropriées.

Enfin, une avancée législative importante est intervenue en juillet 2017. Après quatre années de discussion, le crime de torture a été introduit dans la législation italienne par loi $\mathrm{n}^{\circ} 110 \mathrm{du} 14$ juillet 2017. La torture est désormais un crime condamné par une peine allant de 4 à 10 ans de réclusion au sens de l'art. 613 bis du Code pénal. De même, l'incitation par un agent public à commettre un acte de torture est condamnée par l'art. 613 ter à une peine de réclusion allant de 6 mois à 3 ans. Le CPT salue cette initiative, mais soulève d'importantes failles dans la rédaction du texte. En effet, si le législateur y 
consacre enfin le crime de torture, les dispositions manquent de clarté. Ainsi, pour que le crime de torture soit constitué, il faut qu'il y ait eu des violences et des menaces, mais surtout, que la torture ait été commise par le biais de " plusieurs actes ». Par ailleurs, l'art. 613 ter semble davantage être une circonstance aggravante qu'une infraction pénale autonome. Le CPT, tout comme la doctrine, critiquent largement cette disposition. Il voit dans le choix des termes une potentielle limite à la qualification de crime de torture et un obstacle à la mise en conformité de la législation italienne avec la Convention de New York contre la torture de 1984 et la CEDH. Reste à voir de quelle façon les juges, et sûrement aussi la Cour constitutionnelle, interpréteront ces nouveaux articles.

Comité européen pour la prévention de la torture et des peines ou traitements inhumains et dégradants, Report to the Italian Government on the visit to Italy carried out by the European Committee for the Prevention of Torture and Inhuman or Degrading Treatment or Punishment from 8 to 21 April 2016, CPT/Inf (2017) 23, Strasbourg : Conseil de l'Europe, 8 septembre $2017,69 \mathrm{p}$.

Corte costituzionale, décision $n^{\circ} 22$ du 9 novembre 2016 ; décision $n^{\circ} 17$ du 7 décembre 2016 ; décision $n^{\circ} 20$ du 7 décembre 2016; décision $n^{\circ} 122$ du 8 février 2017 ; décision $n^{\circ} 83$ du 7 mars 2017 ; décision n 76 du 8 mars 2017 ; décision n 179 du 7 juin 2017.

Cour EDH, 2ème Sect., 16 juillet 2009, Sulejmanovic c. Italie, Req. $\mathrm{n}^{\circ}$ 22635/03 ; Cour EDH, 2ème Sect. 8 janvier 2013, Torreggiani et autres c. Italie, Req. nos 43517/09, 46882/09, $55400 / 09,57875 / 09,61535 / 09,35315 / 10$ et $37818 / 10$.

Les Lettres « Actualités Droits-Libertés » (ADL) du CREDOF (pour s'y abonner) sont accessibles sur le site de la Revue des Droits de l'Homme (RevDH) - Contact

\section{NOTES}

1. Corte costituzionale, décision $n^{\circ} 22$ du 9 novembre 2016 ; décision $n^{\circ} 17$ du 7 décembre 2016 ; décision $n^{\circ} 20$ du 7 décembre 2016 ; décision $n^{\circ} 122$ du 8 février 2017 ; décision $n^{\circ} 83$ du 7 mars 2017 ; décision nº 76 du 8 mars 2017 ; décision nº 179 du 7 juin 2017.

2. Cour EDH, 2ème Sect., 16 juillet 2009, Sulejmanovic c. Italie, Req. $n^{\circ}$ 22635/03 ; Cour EDH, 2ème Sect. 8 janvier 2013, Torreggiani et autres c. Italie, Req. nos 43517/09, $46882 / 09,55400 / 09,57875 / 09,61535 / 09,35315 / 10$ et 37818/10.

3. Comité européen pour la prévention de la torture et des peines ou traitements inhumains et dégradants, Report to the Italian Government on the visit to Italy carried out by the European Committee for the Prevention of Torture and Inhuman or Degrading 
Treatment or Punishment from 8 to 21 April 2016, CPT/Inf (2017) 23, Strasbourg : Conseil de l'Europe, 8 septembre 2017, 69 p.

\section{RÉSUMÉS}

Selon l'ancienne Ministre de la Justice italienne, Paola Severino, "nous sommes face à une urgence qui risque de bouleverser le sens même de notre culture juridique, puisque en principe le détenu est privé de ses libertés seulement pour purger sa peine et que ne peut lui être niée sa dignité en tant que personne humaine ». L'Italie fait partie de ces trop nombreux pays qui font face à des difficultés au sein de leurs prisons. À la surpopulation carcérale, s'ajoutent des traitements inhumains et dégradants fréquents. Malgré les efforts de l'Italie pour se conformer aux exigences internationales en la matière, le Comité européen pour la prévention de la torture continue de porter un regard critique sur la situation actuelle au sein des prisons italiennes.

\section{AUTEURS}

\section{YÉRIS NICOLAS}

Étudiante du Master 2 Droit français - droit italien à l'Université Paris Nanterre (en partenariat avec l'Alma Mater Studiorum Università di Bologna) et du Master 2 Propriété industrielle et artistique à l'Université Panthéon-Sorbonne.

\section{MARGAUX ROMANO}

Étudiante du Master 2 Droit français - droit italien à l'Université Paris Nanterre (en partenariat avec la Cattolica del Sacro Cuore di Milano). 